\title{
Paradoxical sensory reactivity induced by functional disconnection in a robot model of neurodevelopmental disorder
}

\author{
Hayato Idei $^{1} \quad$ Shingo Murata $^{2} \quad$ Yuichi Yamashita ${ }^{3, *} \quad$ Tetsuya Ogata $^{4,5, *}$ \\ ${ }^{1}$ Department of Intermedia Studies, Waseda University, Tokyo, Japan \\ ${ }^{2}$ Department of Electronics and Electrical Engineering, Keio University, Tokyo, Japan \\ ${ }^{3}$ Department of Information Medicine, National Center of Neurology and Psychiatry, Tokyo, Japan \\ ${ }^{4}$ Department of Intermedia Art and Science, Waseda University, Tokyo, Japan \\ ${ }^{5}$ Lead Contact \\ *Corresponding authors: yamay@ncnp.go.jp (Yuichi Yamashita), ogata@waseda.jp (Tetsuya Ogata)
}




\begin{abstract}
Hyper- and hyporeactivity to sensory stimuli is a diagnostic feature of autism spectrum disorder and has been reported in many neurodevelopmental disorders. However, the computational mechanisms underlying such paradoxical responses remain unclear. Here, using a robot controlled by a hierarchical recurrent neural network model with predictive processing and a learning mechanism, we simulated how functional disconnection alters the learning process and affects subsequent behavioral reactivity to environmental change. The results show that, through the learning process, functional disconnection between distinct network levels simultaneously lowered the precision of sensory information and higher-level prediction. These changes caused the robot to exhibit sensory-dominated and sensory-ignoring behaviors ascribed to sensory hyperreactivity and hyporeactivity, respectively. Furthermore, local functional disconnection at the sensory processing level similarly induced hyporeactivity due to low sensory precision. These findings suggest a computational explanation for co-existing sensory hyper- and hyporeactivity and insights at various levels of understanding in neurodevelopmental disorders.
\end{abstract}

Keywords Computational psychiatry, Neurodevelopmental disorder, Sensory symptoms, Neurorobotics, Predictive coding, Recurrent neural network, Uncertainty, Disconnection, Learning, Online adaptation 


\section{Introduction}

Autism spectrum disorder (ASD) and other neurodevelopment disorders are characterized by large neurobiological and clinical heterogeneity. Different etiological pathways may lead to the same disorder, and multiple behavioral outcomes can occur within individual patients [I]]. In addition, overlap has been reported in the general characteristics and symptoms of ASD and attention-deficit hyperactivity disorder as well as other mental disorders, such as schizophrenia [2, 3, 4, 5]. The heterogeneous and non-specific nature of the clinical symptoms of these disorders make it difficult to clarify their causes and even to categorize the disorders precisely [6, 7, 1]. In particular, hyper- and hyporeactivity to sensory stimulus are included in the diagnostic criteria for ASD [8] and have been reported across many neurodevelopmental disorders [9]. Despite the phenotypic diversity of ASD, it is estimated that hyper- and hyporeactivity in multiple sensory modalities is present in over $96 \%$ of children with ASD [I0]. Such symptoms emerge over the first few years of life and are to be an early sign of ASD [11, 12, 13, [14]. Unusual sensory reactivity are thought to increase the risk of other problems, such as social impairments and behavioral inflexibility [10, 12], and critically impact the activities of affected individuals over the course of their life. However, the co-existence of unusual paradoxical states, specifically hyper- and hyporeactivity to sensory stimuli, makes it difficult to fully understand the computational and neural underpinnings of ASD.

A computational theory based on a predictive processing framework has been proposed in which neurodevelopmental disorders are associated with unusual reactions to sensory information due to an imbalance between the weight of sensory information and that of prior belief (prediction) [15, 16]. According to the predictive processing framework [17, 18], the brain has an internal model of the world and constantly generates predictions about future sensory inputs. How the brain reacts to sensory information is explained as a hierarchical inferential process of matching internally generated top-down predictions to incoming bottom-up sensory information. The balance between top-down predictions and bottom-up sensory information relies on the estimated precision, or the confidence, of each piece of information and determines the extent to which the brain changes its internal model based on generated prediction errors between prior predictions and actual sensory inputs. In that framework, excessively high precision of sensory information or excessively low precision of prediction characterizes perceptions and behaviors that are dominated by strong bottom-up sensory information with a weak influence of top-down predictions. The consequent sensory overload has been proposed to explain symptoms of ASD, such as hyperreactivity to sensory stimulus and difficulties in social contexts [19, 20, 21]. However, the aberrant precision theory alone has not been able to fully explain the co-existence of hyper- and hyporeactivity to sensory stimuli [22].

Brain imaging studies and theoretical studies have suggested that neurodevelopmental disorders involve reduced functional (effective) connectivity between the frontal cortex (associated with top-down predictive processes) and the posterior parts of the brain (associated with bottom-up sensory processes) [23, 24, 25, 26]. The functional disconnection hypothesis posits that disruption of system-level functions in the brain might be caused by cellular defects in modulating synaptic efficacy [27, 28]. ASD has been regarded as a developmental disconnection 
syndrome [29], with altered functional connectivity suggested to begin as early as the first year of life [30, 31, 32].

However, effects of functional disconnection on learning processes and the mechanisms leading to symptoms remain unclear.

Using a neurorobotics framework with hierarchical predictive processing and a learning mechanism, this study aimed to investigate the mechanistic relationship among developmental functional disconnection, aberrant precision, and unusual sensory reactivity in neurodevelopmental disorders. Previous computational simulation studies of psychiatric symptoms have largely been limited to numerical simulations of perceptual outcomes [33, 34, 35]. For modeling behavior-level symptoms observed in real life and clinical practice, the use of a physical robot might be useful for confirming whether a constructed model of psychiatric disorder can actually produce behavioral symptoms in response to the real world [36, 37, 38]. In particular, sensory uncertainty generated from dynamic brain-body-environment interactions in the real world is difficult or even virtually impossible to reproduce in a numerical simulation. In this study, a simple robot experiment was designed to test the learning of multiple visuomotor patterns and subsequent adaptive behaviors in response to changing visual information. Through the learning phase, a robot controlled by a hierarchical neural network estimated the uncertainty (inverse precision) of sensory information that is naturally generated through physical interaction between the robot's body and an external object. Then, in the adaptation phase, the robot reacted to environmental change via updating of higher-level predictions by minimizing prediction error weighted by the precision of the sensory information. Through the robot experiment, we show how functional disconnection during the learning process alters the precisions of sensory information and higher-level prediction, and the resulting impact on behavioral reactivity to environmental change.

\section{Results}

\section{Computational framework and task setting}

We used a humanoid robot controlled by a recurrent neural network (RNN) model. The model used was a stochastic continuous time RNN with parametric bias (S-CTRNNPB) (Figure $\mathrm{Wa}$ ), which is a type of hierarchical predictive processing model with learning and online adaptation mechanisms [39, 40]. During task execution by the robot, the hierarchical neural network receives current sensory inputs $x_{t}$ comprising 2-dimensional vision of an external object and 8-dimensional proprioception of joint angles. The sensory inputs are propagated to lower-level neurons with recurrent connections and dynamically integrated with top-down signal from higher-level neurons. Here, activities of lower-level neurons represent short-term sensory processing while those of higher-level neurons represent a goal-directed signal or higher-level prediction. Based on the integrated information, the network predicts mean $y_{t}$ and variance $v_{t}$ (inverse precision) of future sensory inputs. The robot moves its joint angles according to the mean proprioceptive prediction. In addition, synaptic weights $w$, the internal state of higher-level neurons, and other parameters are optimized to minimize negative $\log$-likelihood $L_{t}$, which is equivalent to precision-weighted prediction error. 
a

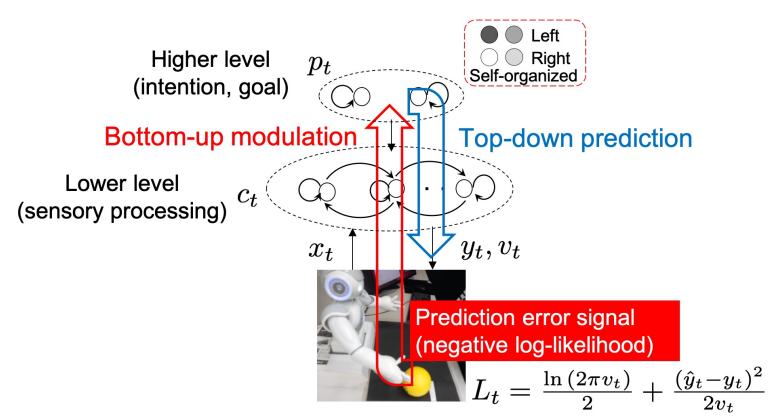

C

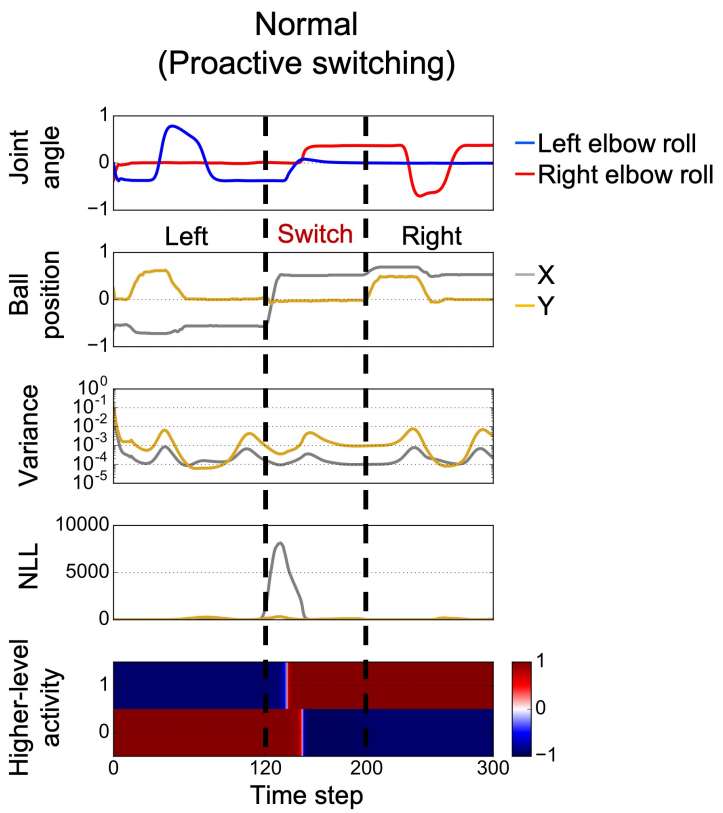

b

\section{Learning phase}

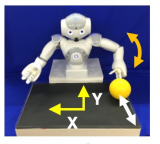

Left

Adaptation phase

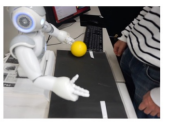

Steps 0-100

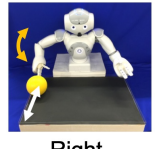

Right

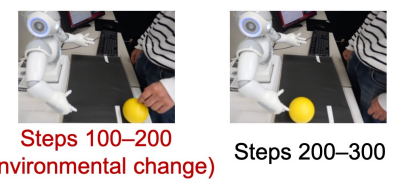

d

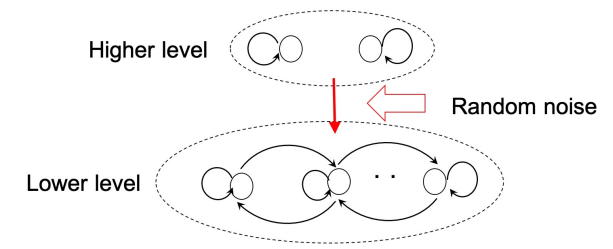

Local functional disconnection

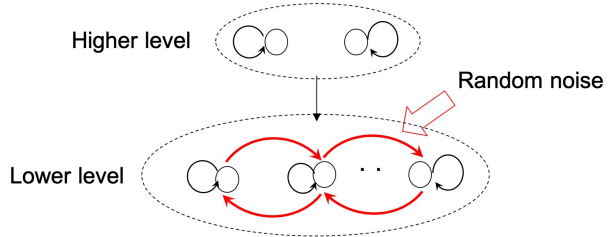

Figure 1: Neurorobotics framework and task design. (a) Top-down and bottom-up interactions in a neural network-controlled robot. A hierarchical recurrent neural network generates top-down predictions about the next sensory states $y_{t}$ and their variance $v_{t}$ (uncertainty) based on current sensory inputs $x_{t}$, lower-level activity $c_{t}$ (sensory processing), and higher-level activity $p_{t}$ (a goal-directed signal, i.e., intention). Synaptic weights and the activities of higher-level neurons are optimized in a bottom-up manner by minimizing negative log-likelihood (precision-weighted prediction error) $L_{t}$ calculated using predictions about sensory states $y_{t}$, their variance $v_{t}$, and actual target sensory states $\hat{y}_{t}$. (b) The robot first learned visuomotor patterns for two ballpass behaviors in the learning phase. Then, the robot performance was tested through a real-time behavior switching task in the adaptation phase. (c) Example of generated time series data through proactive behavior switching by the robot controlled by normal trained network in the adaptation phase. During time steps 0-100, the robot performed a learned ball interaction with an experimenter. The ball position was switched during time steps 100-200 (at time step 120), which generated large prediction error signals. The robot flexibly switched its higher-level activity (prediction) toward minimizing generated error signals and proactively generated the appropriate behavioral pattern during time steps 200-300. "Joint angle": predicted joint angle (2 of 8 joint angles are shown). "Ball position": vision inputs of ball position. "Variance" indicates the prediction of variance in vision. "NLL": negative log-likelihood (precision-weighted prediction error) for vision after updating the activity of higher-level neurons.(d) Simulation of functional disconnection with addition of slight random noise to synaptic weights through the learning process. Long-range functional disconnection indicates disrupted connections between the higher- and lower-level neurons while local functional disconnection indicates disrupted connections within lower-level neurons.

In the learning phase, the robot learned two types of ball-pass behaviors, namely, "left" and "right", in which the robot waited to receive the ball from the experimenter and then returned it (Figure $\mathbb{b}$ ). By repeatedly updating the parameters, the hierarchical network learns to reproduce target visuomotor sequences that were recorded in advance 
during human-robot interaction. Importantly, the associations between specific patterns of higher-level activity and corresponding target visuomotor sequences are self-organized. In this sense, higher-level neural activity can be regarded as a higher-level prediction about the ongoing context or "intention" (or goal) of the robot.

Next, in the adaptation phase, the trained robot reacted to an environmental change and updated its higher-level

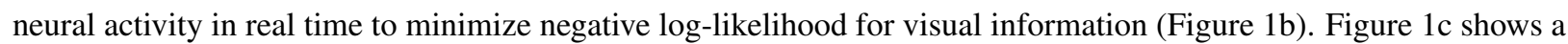
behavior switch performed by a robot controlled by a normal network. During the first 100 steps, the robot generated a learned behavioral pattern, where the sensory experiences (e.g., the timing and speed of the ball) were slightly different from the learned experiences due to the intrinsic uncertainty of the environment. Then, the ball position was unexpectedly switched by the experimenter during time steps 100-200 (the switch occurred at time step 120). The environmental change generated large prediction error signals, but the robot flexibly switched its higher-level neural activity and generated the appropriate behavior for the new situation during time steps 200-300. In this way, the hierarchical interaction of top-down prediction and bottom-up modulation enabled the robot to switch its intention and behavior in response to the environmental change. This seemingly intentional behavior switching of the robot was regarded as a "proactive switch" in this study.

\section{Long-range functional disconnection simultaneously induced sensory-dominated and sensory-ignoring behaviors}

To test the disconnection hypothesis of disrupted top-down and bottom-up interactions in the learning process in neurodevelopmental disorder, we simulated long-range functional disconnection between distinct levels of the hierarchical network (Figure Пd). Specifically, synaptic weights of connections between the higher-level and the lower-level neurons were slightly modified by adding random noise in the learning process. These altered weights are taken to represent altered synaptic connectivity in the brain.

As the result of long-range functional disconnection through the learning process, the robot exhibited abnormal paradoxical behaviors, specifically sensory-dominated behavior and sensory-ignoring behavior, in the adaptation phase. In the long-range disconnection condition, the robot generated a learned behavior before the environmental change (time steps $0-100$ ), but prediction error signals were attenuated by overestimated sensory variance, leading to invariant higher-level neural activity (prediction) throughout the trial. Despite the invariant higher-level prediction, the robot could sometimes exhibit overtly successful behavior switching (Figure $\llbracket a$ ). This means that the robot's action switching was automatically elicited by sensory inputs without proactive control. The sensory-dominated behavior of the robot can be considered to be a consequence of the greatly weakened influence of higher-level prediction compared with that of sensory information. We call such sensory-dominated behavior "reactive switching." On the other hand, the robot controlled by the same neural network could also exhibit a failure of behavior switching due to the invariant higher-level prediction (Figure $2 \mathrm{~b}$ ). The sensory-ignoring behavior can be considered to be a consequence of the overly weak influence of sensory information compared with that of higher-level prediction. We call such sensory-ignoring behavior "perseverative error." 
a

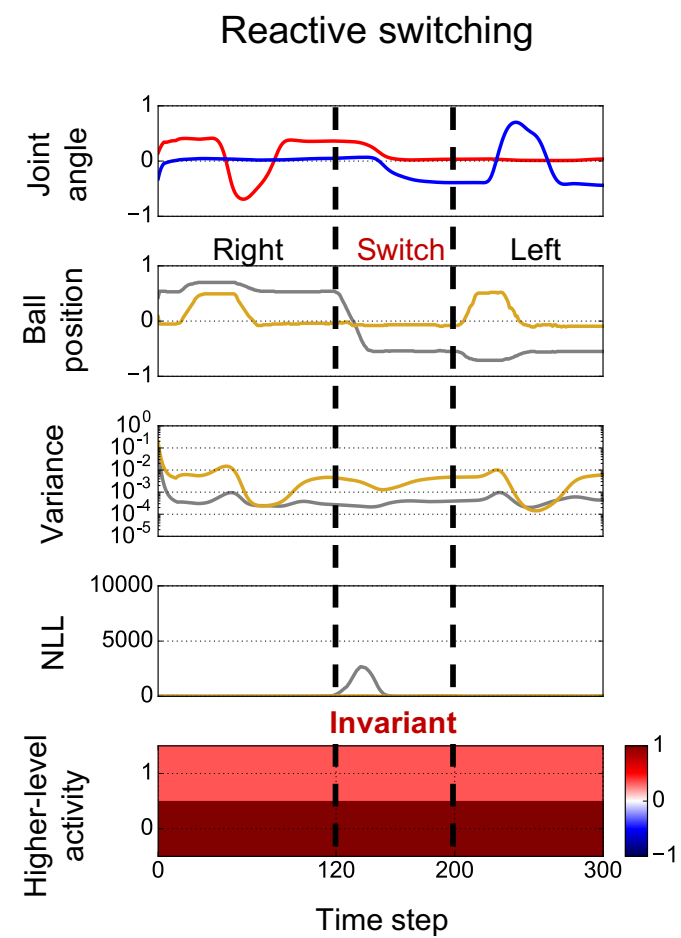

b

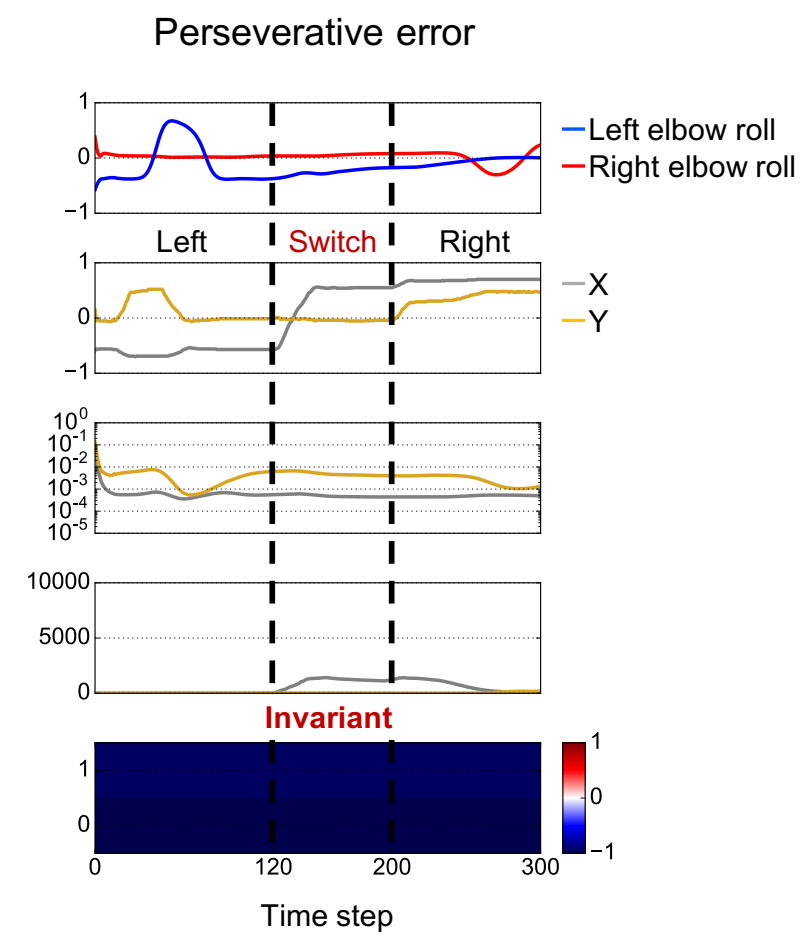

Figure 2: Unusual paradoxical behaviors simultaneously induced by long-range functional disconnection. (a) Example of sensorydominated behavior (reactive switching) observed in the robot controlled by network with long-range functional disconnection $\left(k_{l o n g}=0.2\right)$. The higher-level neural activity (prediction) was invariant throughout the trial because prediction error signals were strongly attenuated by overestimated sensory variance. However, the robot could successfully switch its behavioral pattern by using only sensory inputs without intentional control. This suggests that the robot's behavior was characterized by sensory hyperreactivity due to the very low precision of higher-level prediction. (b) Example of sensory-ignoring behavior (perseverative error) in the robot controlled by the network with long-range functional disconnection $\left(k_{\text {long }}=0.2\right)$. As with the reactive behavior, higher-level prediction of the robot was invariant throughout the trial due to the attenuated prediction error signals arising from overestimated sensory variance. In this case, however, the robot failed to switch its behavioral pattern despite performing appropriate interactions before the environmental change. This suggests that the perseverative behavior was characterized by sensory hyporeactivity due to the very low precision of sensory information. "Joint angle" indicates the prediction of joint angle ( 2 of 8 joint angles are shown). "Ball position" indicates vision inputs of ball position. "Variance" indicates the prediction of variance in vision. "NLL" indicates negative log-likelihood (precision-weighted prediction error) for vision after updating higher-level activity. "Higher-level activity" indicates the activity of higher-level neurons.

The robot's performance under varying severity of long-range disconnection $\left(k_{\text {long }}=0,0.2,0.4\right)$ is summarized in Figure Bla and b. We obtained results for 10 trained neural networks with different initial synaptic weights. Sixty trials were performed for each network condition $k_{l o n g}$ ( 6 trials per network). We evaluated whether the robot's behaviors before and after the environmental change were successful by comparing the acquired time series data with target training data (see Methods section in more detail).

Figure Bla shows the success rate before environmental change (time steps 0-100) in each network condition $k_{\text {long }}$. The graph indicates that under all conditions, the robot could perfectly generalize to performing learned behavioral patterns per se. Figure [3b shows the changes in the robot's behavior after environmental change (time steps 200-300). Observed behavioral characteristics after environmental change were divided into four types 
a

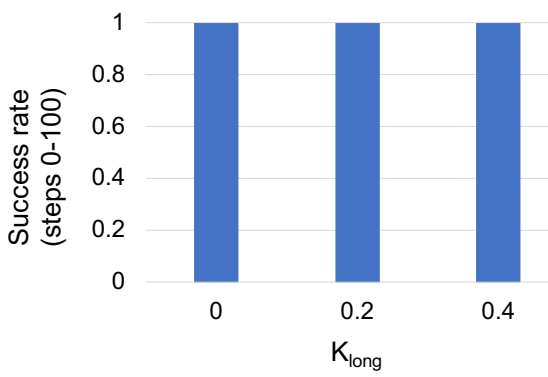

C

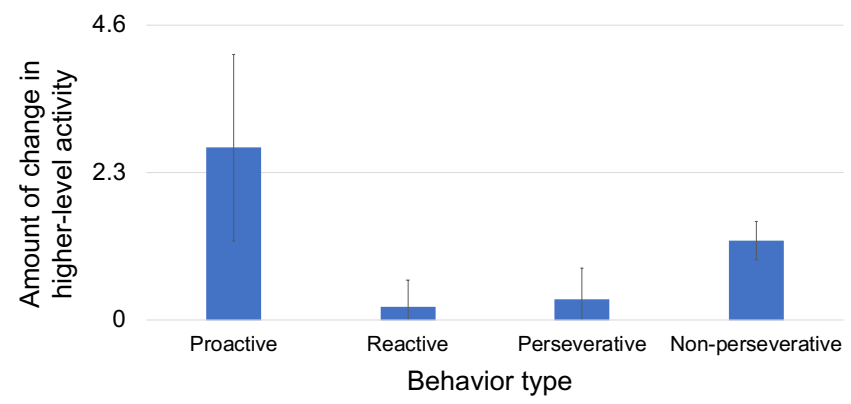

b

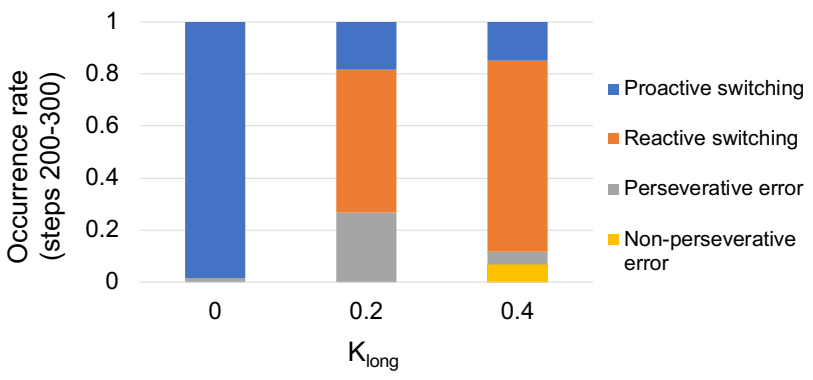

d

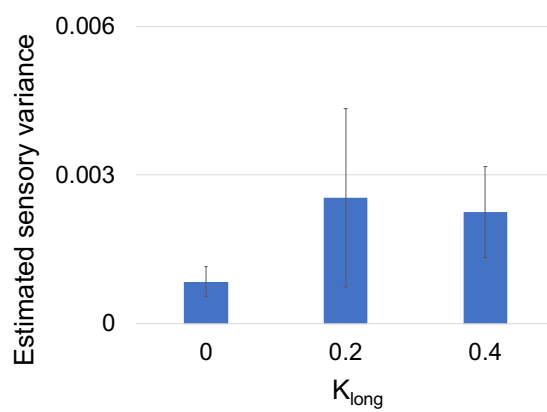

Figure 3: Quantitative analyses of overt and internal characteristics of the robot's performance varying with severity of long-range functional disconnection $\left(k_{l o n g}\right)$. (a) Success rates in the ball-pass interaction before environmental change (time steps 0-100). The values are averages for 10 trained networks ( 6 trials per network). Under all conditions, the robot could perfectly perform learned behaviors before the environmental change. (b) Occurrence rates of each type of behavior after environmental change (time steps 200-300). The values are averages for 10 trained networks. Long-range functional disconnection induced reactive switching and perseverative error. A shift from perseverative behavior to reactive switching was observed as the disconnection became more severe. (c) Total change in higher-level neural activity during environmental change (time steps 100-200) for each behavior type. In reactive switching and perseverative error, the changes in higher-level activity (prediction) were greatly reduced. There were 79 proactive switches, 77 reactive switches, 20 perseverative errors, and 4 non-perseverative errors in 180 trials. (d) Levels of estimated sensory variance during environmental change (time steps 100-200). The values are averages for 10 trained networks. Long-range functional disconnection induced increased estimated sensory variance (decreased sensory precision). Error bars indicate SD.

("proactive switching," "reactive switching," "perseverative error," and "non-perseverative error"). Proactive switching refers to successful behavior by updating higher-level prediction activity while reactive switching refers to overtly successful behavior with weak influence of higher-level prediction activity. Perseverative error means a failure due to a deficit in switching higher-level activity while non-perseverative error means a failure due to an irrelevant change in higher-level activity. According to one-way analysis of variance (ANOVA), the frequency of reactive switching was significantly different among the three network conditions $(F(2,27)=30.45, p<0.001)$. In addition, post hoc multiple comparisons adjusted using the Shaffer method [41] revealed that the frequency of reactive switching was significantly higher for $k_{l o n g}=0.2,0.4$ than for the normal condition $\left(k_{l o n g}=0\right)$ $(p<0.001)$. This suggests that long-range functional disconnection consistently increased the frequency of reactive switching. The frequency of perseverative error was also significantly different among the three conditions $(F(2,27)=5.06, p=0.014$,one-way ANOVA $)$. However, according to the post hoc Shaffer test, the frequency of perseverative error was significantly different between $k_{\text {long }}=0$ and $k_{\text {long }}=0.2(p=0.0068)$ and between $k_{\text {long }}=0.2$ and $k_{\text {long }}=0.4(p=0.017)$, indicating that the frequency was highest in the mild condition 
$\left(k_{l o n g}=0.2\right)$ rather than in the severe condition $\left(k_{l o n g}=0.4\right)$. These results suggest that long-range functional disconnection could induce sensory-dominated behavior and sensory-ignoring behavior, but the balance was shifted to sensory-dominated behavior as the disconnection became more severe.

To quantitatively confirm the internal characteristics underlying the abnormal behaviors, the total change in higher-level neural activity and the estimated sensory variance (inverse sensory precision) were analyzed. Figure Bic shows the total change in higher-level neural activity during - environmental change (the sum of the changes during time steps 100-200) for each of the four behavior types; the value is the average of all trials for each behavior type. There were 79 proactive switches, 77 reactive switches, 20 perseverative errors, and 4 non-perseverative errors out of all 180 trials. The total change in higher-level activity was significantly different among proactive switching, reactive switching, and perseverative error $(F(2,173)=120.87, p<0.001$, one-way ANOVA). In addition, the total change in higher-level activity was significantly lower for reactive switching and perseverative error than for proactive behavior ( $p<0.001$, post hoc Shaffer test). This indicates that reactive switching and perseverative error were characterized by a small total change in higher-level neural activity. Figure Bld shows the estimated level of sensory variance during environmental change (time steps 100-200) in each network condition $k_{\text {long }}$. The values are averages for 2 visual dimensions, 100 time steps, 6 trials, and 10 trained networks. The estimated sensory variance (inverse sensory precision) was significantly different among the three network conditions ( $\left.k_{l o n g}=0,0.2,0.4\right)$ $(F(2,27)=5.32, p=0.011$, one-way ANOVA). In addition, according to the post hoc Shaffer test, the estimated sensory variance was significantly different between $k_{\text {long }}=0$ and $k_{\text {long }}=0.2(p=0.0051)$ and between $k_{l o n g}=0$ and $k_{\text {long }}=0.4(p=0.018)$, indicating that the variance was increased by long-range functional disconnection. These results suggest that increased sensory variance (decreased sensory precision) and the consequent invariant higher-level neural activity resulted in the abnormal behaviors.

\section{Local functional disconnection similarly induced sensory-ignoring behavior}

We also investigated the effects of local functional disconnection within lower-level neurons, which is assumed to represent functional disconnection within brain regions associated with sensory processing (Figure Wd).

Local functional disconnection induced overestimated sensory variance and consequently sensory-ignoring behavior, whereas sensory-dominated behavior was not observed. Figure [la, b, and c respectively show the robot's performance before environmental change (time steps 0-100), behavioral characteristics after environmental change (time steps 200-300), and the estimated level of sensory variance during environmental change (time steps 100-200) for two severity levels of local functional disconnection $\left(k_{\text {local }}=0,0.015,0.03\right)$. The values are averages of 60 trials in each $k_{\text {local }}$ network condition (i.e., 6 trials for each trained network). Under all conditions, the robot's performance in generating learned behaviors remained intact $(F(2,27)=0.70, p=0.51$, one-way ANOVA). However, the frequency of perseverative error was significantly different among the three conditions $(F(2,27)=5.34, p=0.011$, one-way ANOVA). According to the post hoc Shaffer test, the frequency of perseverative error was significantly different between $k_{\text {local }}=0$ and $k_{\text {local }}=0.03(p=0.0049)$ and between $k_{\text {local }}=0.015$ and $k_{\text {local }}=0.03$ 
a

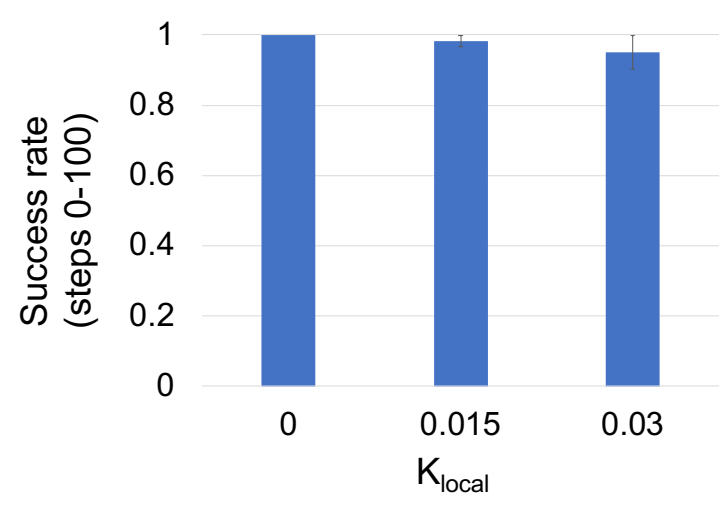

C

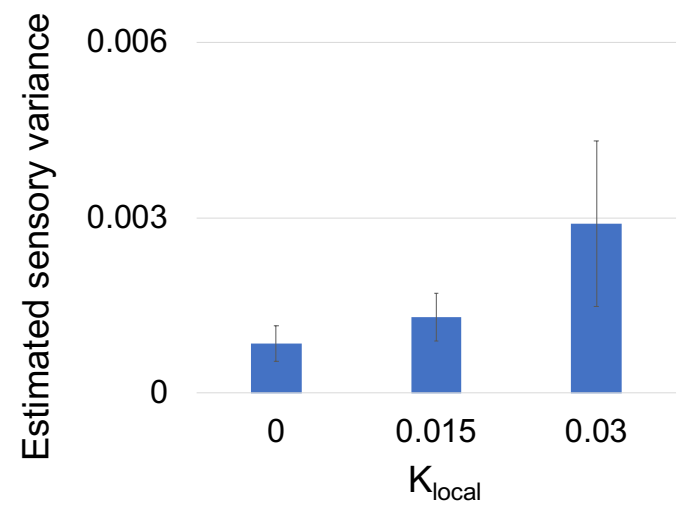

b

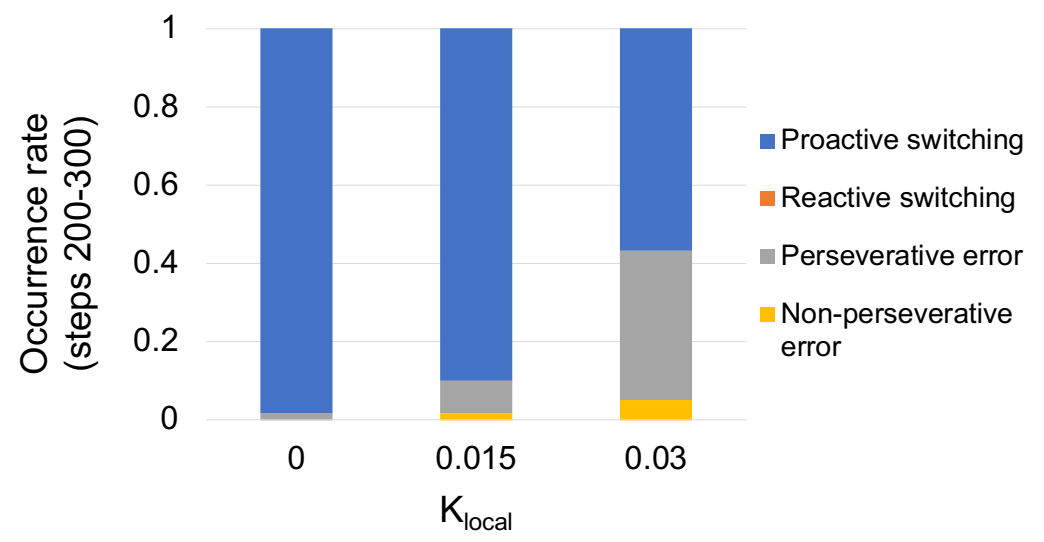

Figure 4: Quantitative analyses of overt and internal characteristics of robot's performance with varying severity of local functional disconnection $\left(k_{\text {local }}\right)$. (a) Success rates in the ball-pass interaction before environmental change (time steps $0-100$ ). The success rates under all conditions were $95 \%$ or higher. (b) Occurrence rates of each behavior type after environmental change (time steps 200-300). Increased perseverative error was observed in the severe condition $\left(k_{l o c a l}=0.03\right)$. However, unlike the case of long-range functional disconnection, reactive switching was not observed. (c) Levels of estimated sensory variance during environmental change (time steps 100-200). As with the case of long-range functional disconnection, increased estimated sensory variance (decreased sensory precision) was observed. Six trials were performed by each trained network (i.e., 60 trials in each $k_{\text {local }}$ condition). All results are reported as averages for 10 trained networks. Error bars indicate SD.

( $p=0.018$ ), indicating that the frequency increased as local functional disconnection became more severe. In addition, the estimated sensory variance was significantly different among the three conditions $(F(2,27)=13.97$, $p<0.001$, one-way ANOVA). The estimated sensory variance was significantly different between $k_{\text {local }}=0$ and $k_{\text {local }}=0.03$ and between $k_{\text {local }}=0.015$ and $k=0.03$ (both $p<0.001$, post hoc Shaffer test), indicating estimated sensory variance was increased as local functional disconnection become more severe. These results suggest that sensory-ignoring behavior due to overestimated sensory variance (low sensory precision) was induced by local functional disconnection as well as long-range functional disconnection. 


\section{Difference in higher-level neural representation}

To investigate why the behavioral differences between the conditions of long-range and local functional disconnections were observed, we analyzed how the two learned visuomotor patters were represented in higher-level neural activity. The number of higher-level neurons in the used neural network was two, thus, each behavior can be considered to be represented in a 2-dimensional space. Heat maps in Figure $\square$ show the prediction error for training

a

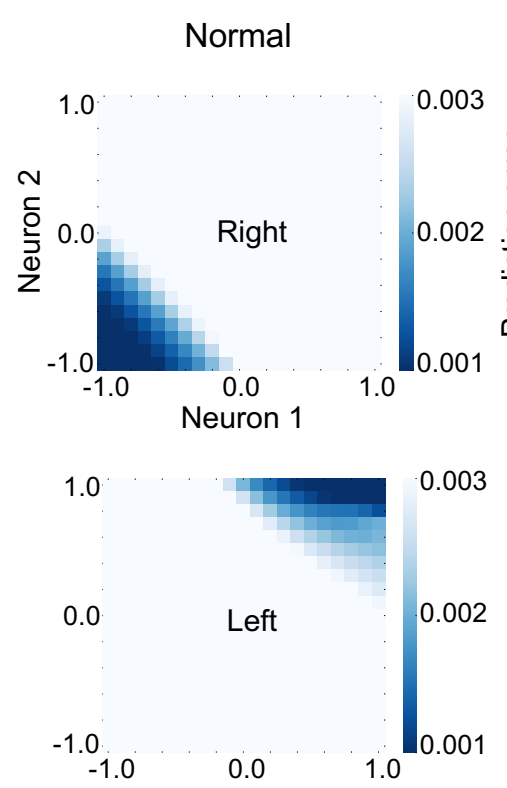

b
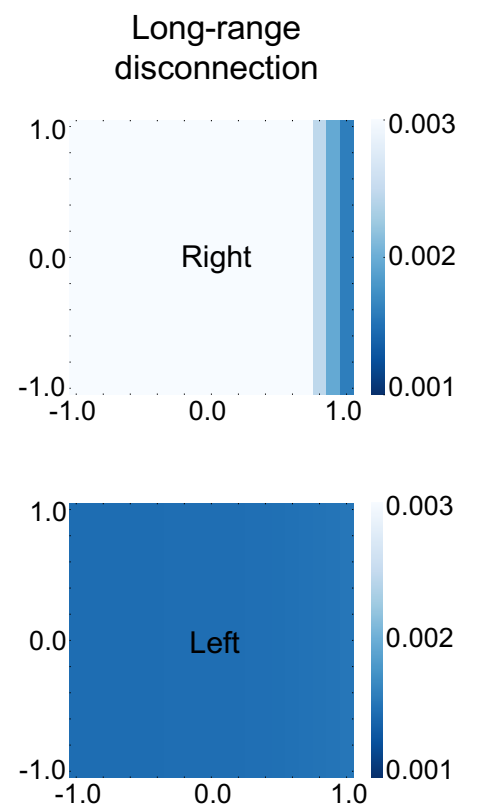

C

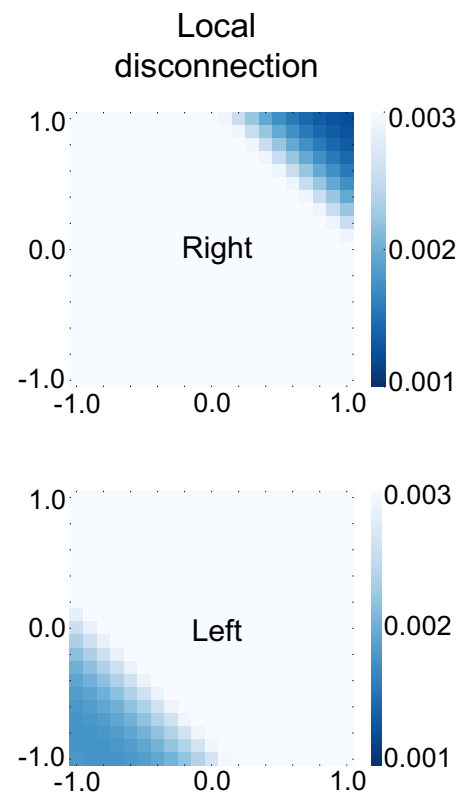

d Long-range disconnection

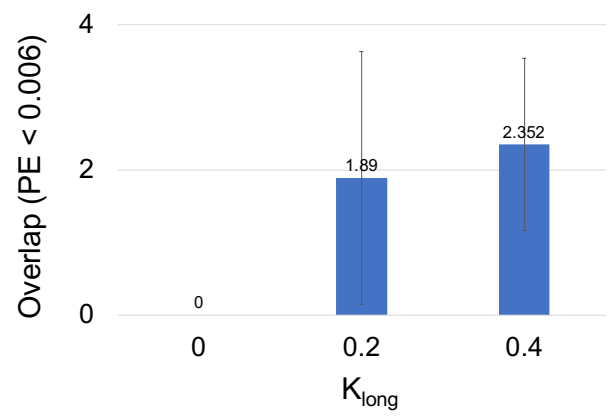

e

Local disconnection

Figure 5: Changes in higher-level neural activity that encodes learned visuomotor patterns. (a, b, c) Heat maps show levels of prediction error for training data under varying higher-level neural activity. Upper and lower panels show prediction errors for training data of left and right ball-pass behaviors, respectively. The prediction errors are shown as averages for all sensory dimensions, time steps, and training datasets. Long-range functional disconnection $\left(k_{\text {long }}=0.2\right)$ loosened the higher-level neural representations and there was overlap of neural activities encoding each behavioral pattern, suggesting low precision of higherlevel predictions. On the other hand, as in the normal condition, such overlap was not observed in the case of local functional disconnection $\left(k_{\text {local }}=0.03\right)$. "Neuron 1 " and "Neuron 2" indicate the higher-level neurons. (d, e) The overlap area of higherlevel activities for which the calculated training errors for both left and right ball-pass behaviors were lower than 0.006. The graphs quantitatively confirms the increased overlap in the case of long-range functional disconnection and no overlap in the case of local functional disconnection. Reported values are the mean \pm SD for 10 trained networks. 
data of left (upper) or right (lower) ball-pass behaviors varying with higher-level neural activity. Prediction errors are averages over all sensory dimensions, time steps, and training datasets. The deep blue regions in the heat maps, where the prediction errors for the corresponding training data are small, can be considered to encode learned behaviors. From Figure 5la and c, higher-level neural activities encoding the distinct behaviors were separated in normal condition and local disconnection condition. However, there was overlap between neural activities encoding left ball-pass behavior and those encoding right ball-pass behavior in the condition of long-range functional disconnection (Figure $5 \mathrm{~b}$ ). In particular, Figure $5 \mathrm{~b}$ shows that left ball-pass behavior could be generated with any higher-level neural activities, suggesting highly weak influence of higher-level prediction on the behavioral generation. In that network, behavior switching from right ball-pass behavior to left ball-pass behavior could be achieved without changes in higher-level prediction while behavior switching from left ball-pass behavior to right ball-pass behavior might require changes in higher-level prediction. This characteristic in higher-level neural representation and overestimated sensory variance was considered to jointly cause the simultaneous generation of sensory-dominated and sensory-ignoring behaviors in a robot as shown in Figure \.

Figure 51 shows the overlap of higher-level neural activities, varying with the severity of long-range functional disconnection $k_{\text {long }}$. The overlap was defined by the area of higher-level neural activities for which the calculated prediction errors were less than 0.006 for training data of both left and right ball-pass behaviors. The values are averages for 10 networks for each condition $\left(k_{l o n g}=0,0.2,0.4\right)$. The overlap was significantly different among the three conditions $(F(2,27)=9.45, p<0.001$, one-way ANONA). In addition, according to the post hoc Shaffer test, the overlap was significantly different between $k_{\text {long }}=0$ and $k_{\text {long }}=0.2(p=0.0027)$ and between $k_{\text {long }}=0$ and $k_{\text {long }}=0.4(p<0.001)$, indicating that the overlap are became larger as long-range functional disconnection became more severe. This might suggest weakened precision or influence of higher-level prediction might underlie a frequency shift from sensory-ignoring behavior to sensory-dominated behavior in the adaptation phase. In contrast, in the condition of local functional disconnection, no overlap of higher-level neural activities encoding the left and right ball-pass behaviors was observed (Figure \$e). The difference in higher-level neural representation might explain the behavioral differences induced by long-range and local functional disconnections.

\section{Difference in development of synaptic weights}

Finally, we analyzed changes in the development of synaptic weights induced by functional disconnections. Figure Ga-c show the distributions of weights of local connections (upper) and long-range connections (lower) after learning. The probabilities of the weights are averaged over 10 trained networks under all network conditions. As shown in Figure $6 \mathrm{~b}$, long-range functional disconnection led to narrower distribution of synaptic weights, especially in long-range connections, indicating that there were many weak synaptic weights compared with the normal condition. Figure Gc shows that local functional disconnection also led to a narrower distribution of weights of local connections, but there was no big difference in long-range connections compared with the normal condition. 


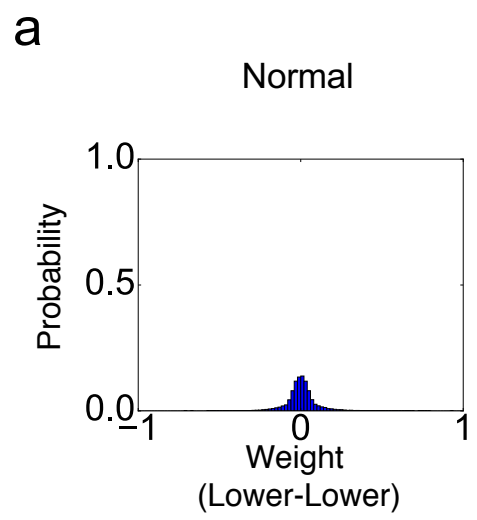

b
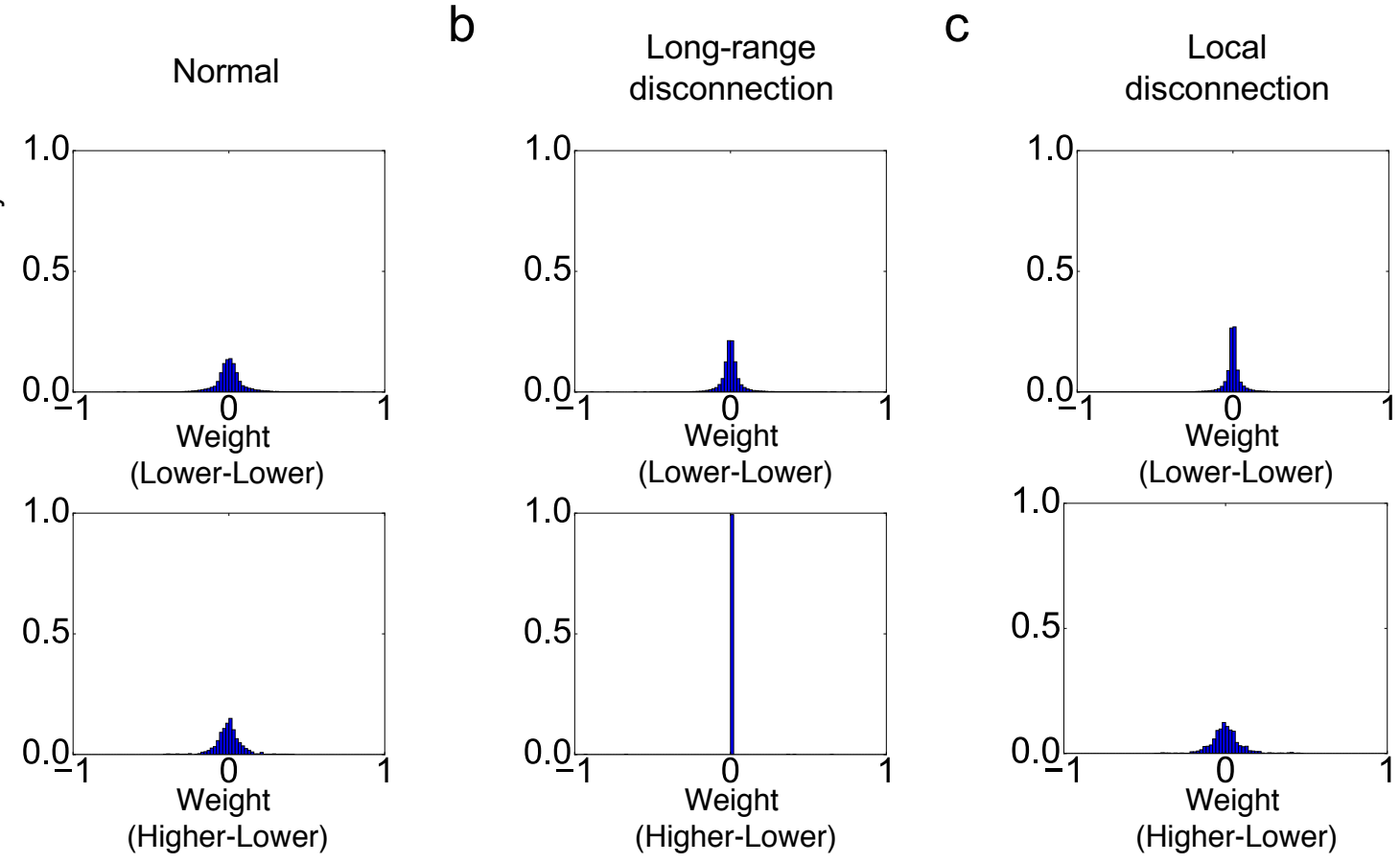

Figure 6: Changes in development of synaptic weights. (a, b, c) Distribution of after learning in each condition. Upper and lower panels respectively correspond to synaptic weights of local connections within the lower-level neurons and long-range connections between the lower- and higher-level neurons. In the case of long-range functional disconnection $\left(k_{\text {long }}=0.2\right)$, long-range connections were substantially weakened (few connections were functioning) and local connections were also slightly weakened compared with the normal condition. In the case of local functional disconnection $\left(k_{l o c a l}=0.03\right)$, local connections were weakened compared with the normal condition, but long-range connections were not altered. The results are shown as the average of 10 networks with different initial weights. 


\section{Discussion}

Using a neural network-controlled robot with hierarchical predictive processing and a learning mechanism, this study investigated the effects of functional disconnection on learning and subsequent behavioral reactivity to environmental change. The robot first learned multiple visuomotor patterns of ball-pass behaviors based on a mechanism of minimizing negative log-likelihood (precision-weighted prediction error). Learning resulted in lower-level and higher-level neural activities of the hierarchical neural network respectively encoded the short-term sensory processing and higher-level prediction about the ongoing context. Then, the trained robot was tested via real-time interaction with an experimenter, during which the robot was required to adapt to environmental changes by updating its higher-level neural activity (prediction) based on minimizing the negative log-likelihood. When controlled by a normal network, the robot exhibited proactive behavior switching by flexibly changing own higher-level prediction in response to unpredictable environmental changes. In contrast, long-range functional disconnection between distinct levels of the hierarchical network simultaneously caused the robot to exhibit unusual paradoxical sensory behaviors, specifically sensory-dominated and sensory-ignoring behaviors. In sensory-dominated behavior, the robot displayed behavior switching depending on only sensory inputs without changes in its higher-level prediction, suggesting hyperreactivity to sensory information due to substantially weakened higher-level prediction. In sensory-ignoring behavior, the robot could not switch its behavioral pattern and exhibited invariant higher-level prediction, suggesting hyporeactivity to sensory information due to very low sensory precision. Such hyporeactivity to sensory information was also induced by local functional disconnection at the level of sensory processing. The proposed computational mechanisms and observed characteristics underlying behavioral alterations might provide insights at various levels of understanding in neurodevelopmental disorders.

At the neural level, neuropsychiatric disorders are proposed to be associated with alterations in pruning, the process of eliminating excess synaptic connections [42]. In ASD, a prevailing hypothesis posits excessive synapses due to underpruning [43] which is supported by the discovery of increased head and brain size in children with ASD [44] as well as a postmortem evidence for increased dendritic spine density [45]. However, an alternative hypothesis argues that ASD involves overpruning, especially of long-range connections [46]. Furthermore, a large longitudinal study with 442 infants at high-risk of ASD and 253 low-risk controls reported no overall difference in head circumference growth over the first 3 years of life between the two groups [47]. These conflicting findings can likely be ascribed in part to the heterogeneity of neurodevelopmental disorders. In our study, long-range functional disconnection induced underdeveloped whole synaptic connections, especially in long-range connections. In other words, the number of weak or unwanted connections was larger than under the normal condition. In addition, local functional disconnection induced weak synaptic weights in only local connections. These results suggest that the number of synaptic connections eliminated via pruning might be increased by either long-range and/or local functional disconnection. In an additional analysis, we also simulated the effects of anatomical reduction of synaptic connections on learning and adaptive behavior. In the supporting results, an $80 \%$ reduction in long-range synaptic connections slightly weakened higher-level prediction, although it did not have a large impact on the robot's sensory 
precision or its behavior-level performance (Supplementary Figure 1). On the other hand, an $80 \%$ reduction in local connections induced sensory-ignoring behavior due to low sensory precision but did not alter higher-level neural activity (Supplementary Figure 2). These findings suggest that excessively reduced anatomical connections might also increase the risk of unusual sensory behaviors.

At the cognitive level, previous computational modeling studies have posited that an imbalance between precision of sensory information and precision of prior belief (prediction) is associated with various psychiatric symptoms [19, 20, 21, 115, 137, 48]. Our findings suggest system-level implications for two questions regarding the aberrant precision theory of neuropsychiatric disorders. Firstly, our results provide a possible neural-level factor (i.e., long-rage and local functional disconnection) which induces aberrant precision in a self-organized manner through the learning process. Secondly, our results provide a possible computational mechanism underlying the co-existence of sensory hyper- and hyporeactivity in individuals with neurodevelopmental disorders such as ASD. Specifically, long-range functional disconnection could simultaneously lower the precision of sensory information and higher-level prediction through the learning process. In that network condition, sometimes bottom-up sensory information was excessively strong compared with top-down prediction, but at other times, the opposite was true. Perhaps long-range and local functional disconnections might act in concert even further increasing the risk of imbalance between the precision of sensory information and the precision of higher-level prediction. This view of coexisting precision imbalances in an individual agent might be informative for understanding various symptoms of neuropsychiatric disorders. For example, psychotic symptoms are suggested to be associated with either increased and decreased precision of prior beliefs compared with that of sensory information [49, 48].

At the behavior level, our findings might provide insights for a clinical understanding of unusual sensory reactivity in neurodevelopmental disorders. The co-existence of behavioral hyper- and hyporeactivity to sensory stimulus is a challenging clinical feature of ASD and other neurodevelopmental disorders. A possible explanation for these paradoxical behaviors is that hyporeactivity as well as behavioral inflexibility are a form of "shut-down" in response to sensory overload or a strategy for producing predictable sensations [50]. However, hyporeactivity shifts to hyperreactivity in the first year of life in infants later diagnosed with ASD [11], suggesting that hyporeactivity might not fully be explained as solely a product of hyperreactivity. Here, our results suggest that the unusual paradoxical behavioral reactivity can actually occur as a direct result of cognitive hyper- and hyporeactivity induced by long-range functional disconnection. In particular, as the severity of long-range functional disconnection increased, a frequency shift from sensory-ignoring behavior to sensory-dominated behavior was observed. Given that functional disconnection in ASD is suggested to begin as early as the first year of life [30, 32], this result might partly explain why hyperreactivity in ASD increases in the first years of life [11, 14, 51]. Clinical studies including a meta-analysis have suggested that hyporeactivity in ASD decreases over the first years of life [11, 51, 52], but there are conflicting findings [14]. Perhaps hyporeactivity as a product of hyperreactivity increases as hyperreactivity becomes severe and the brain develops to process complex information and skills. In addition, local functional disconnection at the level of sensory processing, which has been separately suggested to be associated with 
ASD [53, 54], could also increase the risk of hyporeactivity to sensory stimuli. Therefore, behavioral hyporeactivity might be generated from various pathways involving cognitive hyper- and hyporeactivity, leading to the complex clinical course [5]]. These perspectives might also have implications for clinical findings in ASD, which show that hyperreactivity most robustly distinguished between high-risk infants and low-risk controls at 12 months of age but hyporeactivity best distinguished older children with ASD from normally developing controls [51, 14].

In conclusion, this study suggests that alterations in hierarchical predictive processing in the face of environmental uncertainty might be a key for understanding unusual sensory behaviors in neurodevelpmental disorders. In particular, our findings suggest a possible mechanistic relationship among (developmental) functional disconnection, aberrant precision theory, and unusual sensory reactivity. Given that neurodevelopmental disorders exhibit highly heterogeneous clinical features and neurobiological characteristics, the proposed mechanism may be limited to characterizing a specific subtype of neurodevelopmental disorders. However, the various levels of characteristics observed in this study suggest insights into precise mechanisms underlying observed behaviors and brain activity in psychiatric patients and might aid in subgroup detection. A limitation of the neurorobotic framework used in this study is that sensory inputs are partly different between the human body and the robot, especially in interoception. Exploring interoceptive inferences in robots will be helpful for understanding social deficits and altered sense of self in neuropsychiatric disorders. 


\section{Methods}

\section{Neural network model}

The RNN model (S-CTRNNPB) has five groups of neurons: input, context, parametric bias (PB), mean, and variance. Input neurons receive the current sensory information. Context neurons (lower-level neurons) integrate the past history of context activities with current synaptic inputs from input neurons and PB neurons (higher-level neurons). PB neurons receive only their own previous states and serve as a higher-level goal-directed signal. Based on the integrated dynamic information in the network, the S-CTRNNPB generates predictions about the mean and variance (inverse precision) of the next sensory inputs, which are assumed to follow Gaussian distribution. Synaptic weights and internal state of PB neurons are optimized to minimize negative log-likelihood, which corresponds to precision-weighted prediction error. The S-CTRNNPB implements the idea of hierarchical predictive processing in the brain [ [55, 17, 18] and a characteristic of biological neural network in that neural activity is determined by the past history of neural states as well as current synaptic inputs [56, 57]. In this sense, the current model can be considered consistent with the macro-level mechanisms of biological neural systems. However, the current model cannot consider physiological details, such as features of individual spikes and characteristics of individual synapses. Therefore, possible implications of our results are limited to system-level mechanisms of biological neural systems.

\section{Forward prediction}

The neuronal model is based on the conventional firing rate model. The internal state of the $i$ th neuron at time step $t$, denoted as $u_{t, i}^{(s)}(t \geq 1)$, is calculated as

$$
u_{t, i}^{(s)}=\left\{\begin{array}{lc}
u_{t-1, i}^{(s)} & \left(I \in I_{\mathrm{P}}\right), \\
\frac{1}{\tau_{i}}\left(\sum_{j \in I_{\mathrm{I}}} w_{i j} x_{t, j}^{(s)}+\sum_{j \in I_{\mathrm{C}}} w_{i j} c_{t-1, j}^{(s)}+\sum_{j \in I_{\mathrm{P}}} w_{i j} p_{t, j}^{(s)}+b_{i}\right)+\left(1-\frac{1}{\tau_{i}}\right) u_{t-1, i}^{(s)} \quad\left(i \in I_{\mathrm{C}}\right), \\
\sum_{j \in I_{\mathrm{C}}} w_{i j} c_{t, j}^{(s)}+b_{i} & \left(i \in I_{\mathrm{M}}, I_{\mathrm{V}}\right),
\end{array}\right.
$$

where $I_{\mathrm{I}}, I_{\mathrm{C}}, I_{\mathrm{P}}, I_{\mathrm{M}}$, and $I_{\mathrm{V}}$ are index sets of the input, context, PB, mean, and variance neurons, respectively; $w_{i j}$ is the weight of the synaptic connection from the $j$ th neuron to the $i$ th neuron; $x_{t, j}^{(s)}$ is the $j$ th input at time step $t$ of the $s$ th sequence; $c_{t, j}^{(s)}$ is the $j$ th context activity; $p_{t, j}^{(s)}$ is the $j$ th PB activity; $\tau_{i}$ is the time constant of the $i$ th neuron; and $b_{i}$ is the bias of the $i$ th neuron. From equation (1), PB neurons can be regarded as a specific type of context neurons whose time constant is infinite. In this study, we set the initial values of the internal states of the context neurons to zero, and those of the PB neurons are optimized for each target temporal sequence of learning. This indicates that 
differences among multiple target temporal sequences are represented in the activities of PB neurons and the dynamics of context activities. The output of each neuron is calculated using the following activation functions:

$$
\begin{array}{cl}
p_{t, i}^{(s)}=\tanh \left(u_{t, i}^{(s)}\right) & \left(0 \leq t \wedge i \in I_{\mathrm{P}}\right), \\
c_{t, i}^{(s)}=\tanh \left(u_{t, i}^{(s)}\right) & \left(0 \leq t \wedge i \in I_{\mathrm{C}}\right), \\
y_{t, i}^{(s)}=\tanh \left(u_{t, i}^{(s)}\right) & \left(1 \leq t \wedge i \in I_{\mathrm{M}}\right), \\
v_{t, i}^{(s)}=\exp \left(u_{t, i}^{(s)}\right) & \left(1 \leq t \wedge i \in I_{\mathrm{V}}\right) .
\end{array}
$$

\section{Parameter optimization}

The S-CTRNNPB performs parameter optimization via the gradient descent method, aiming to minimize negative log-likelihood. The negative log-likelihood is formally given by

$$
L_{t, i}^{(s)}=\frac{\ln \left(2 \pi v_{t, i}^{(s)}\right)}{2}+\frac{\left(\hat{y}_{t, i}^{(s)}-y_{t, i}^{(s)}\right)^{2}}{2 v_{t, i}^{(s)}}
$$

Here, $\hat{y}_{t, i}^{(s)}$ is the target value of the $i$ th mean neuron corresponding to the $s$ th sequence. Minimizing negative log-likelihood can be regarded as minimizing the precision-weighted (inversely variance-weighted) prediction error.

During learning, parameters are updated, including synaptic weights $w_{i j}$, biases $b_{i}$, and the initial internal states of PB neurons $u_{0, i}^{(s)}\left(i \in I_{\mathrm{P}}\right)$. Parameter optimization is performed by minimizing the sum of the negative log-likelihood over all sensory dimensions, time steps, and sequences as

$$
L=\sum_{s \in I_{\mathrm{S}}} \sum_{t=1}^{T^{(s)}} \sum_{i \in I_{\mathrm{M}}} L_{t, i}^{(s)}
$$

where $I_{\mathrm{S}}$ and $T^{(s)}$ represent the index set and the length of the $s$ th target temporal sequence, respectively. The partial derivative of each parameter, $(\partial L) /(\partial \boldsymbol{\theta})$, can be solved using the back-propagation-through-time method [39, 58].

During the adaptation phase after learning, only the internal states of the PB neurons are updated; other parameters are fixed. In this phase, the negative log-likelihood within a short time window $W$ is summed as

$$
L=\sum_{t^{\prime}=t-W+1}^{t} \sum_{i \in I_{\mathrm{M}}} L_{t^{\prime}, i}^{(s)}
$$

The time window slides as the network time step $t$ is incremented. The internal states of the PB neurons at time step $t-W$ are optimized using the summed negative log-likelihood. The partial derivative of the internal states of PB neurons is also calculated by the back-propagation-through-time algorithm. 
In both the learning and adaptation phases, parameters permitted to be optimized are collected by $\boldsymbol{\theta}$, and $\boldsymbol{\theta}$ at the $n$th epoch is updated by gradient descent on the accumulated negative $\log$-likelihood $L$ :

$$
\begin{array}{r}
\boldsymbol{\theta}(n)=\boldsymbol{\theta}(n-1)+\Delta \boldsymbol{\theta}(n), \\
\Delta \boldsymbol{\theta}(n)=-\alpha \frac{\partial L}{\partial \boldsymbol{\theta}}+\eta \Delta \boldsymbol{\theta}(n-1) .
\end{array}
$$

Here, $\alpha$ is the learning rate and $\eta$ is a coefficient representing the momentum term. In this study, $\alpha$ and $\eta$ are set at 0.0001 and 0.9 , respectively.

\section{Experimental environment}

We used a small humanoid robot, NAO T14 (SoftBank Robotics, Paris, France), that has a body corresponding to only the upper half of the human body. The robot was placed in front of a workbench and carried out a ball-playing interaction with an experimenter standing at the opposite side. The robot's action involved only movements of the arms, with 4 degrees of freedom for each arm ( 2 degrees for the shoulders and 2 for the elbows). In addition, a camera installed in the robot's mouth obtained the center of gravity coordinates for a yellow ball, which corresponds to the 2-dimensional visual inputs for ball position. Using the minimum and maximum values of each sensory input, the values of joint angles and the ball position were normalized to range from -0.8 to 0.8 . During task execution, the robot received sensory state inputs every $100 \mathrm{~ms}$. The dimensions of the workbench and diameter of the ball were approximately $45 \times 5 \times 30 \mathrm{~cm}$ and $9 \mathrm{~cm}$, respectively.

\section{Training}

The neural network was trained by predictive learning using target perceptual sequences recorded in advance. The target sequences were recorded while the robot repeatedly performed each ball-pass interaction with an experimenter, where the arm movement was generated exactly by following preprogrammed trajectories instead of the ones generated by the neural network model. Each sequence of the two behavioral patterns was obtained as a sequence of 10-dimensional vectors (8-dimensional proprioception of joint angles and 2-dimensional vision of ball position). Three training sequences were prepared for each behavioral pattern. The time length of the sequences for left ball-pass behavior was 905 time steps (10 cycles), and that for right ball-pass behavior was 835 time steps (10 cycles). The neural network learned to reproduce the sequences of training data. With randomly initialized parameters, the neural network initially produces random sequences. However, by repeating parameter updates to minimize the negative log-likelihood, the neural network comes to reproduce visuomotor sequences with the same stochastic properties as the training data. In addition, the associations between a particular pattern of target sequence and specific internal states of PB neurons were self-organized. 


\section{Real-time adaptation}

In the adaptation phase, the robot's higher-level prediction (PB activity) was first set to a certain state corresponding to a learned behavior and the robot performed the corresponding interaction with the experimenter. Then, an environmental change was unpredictably introduced by the experimenter by changing the ball position. The goal of the robot was to flexibly recognize the environmental changes using visual observations. The real-time adaptation process during the task execution of the robot was performed based on an interaction between top-down prediction generation and bottom-up parameter modulation. In the top-down prediction generation process, the network generated a temporal sequence corresponding to time steps from $t-W+1$ to $t$, based on the sensory inputs at time step $t-W+1$ and the constant PB activity. The visuomotor sequence was generated by a closed-loop process, using the predicted mean values of sensory states at each time step as inputs to the next time step. The initial inputs for joint angles at time step $t-W+1$ were the generated mean predictions at time step $t-W$, and the vision states at time step $t-W+1$ are the vision data captured by the camera. In the bottom-up modulation process, the negative log-likelihood at each time step within time window $W$ was calculated from the vision-state predictions, variance, and actual observations. The PB activity (higher-level prediction) was updated to minimize the summed negative log-likelihood. A temporal sequence within the time window was re-generated using the updated PB activity. After repeating these top-down and bottom-up processes for a certain duration, the network generated predictions for time step $t+1$; the predicted joint angles were sent to the robot as the target for the next joint positions. This procedure, in which the recognition and prediction in the past are reconstructed from the current sensory information, is a "postdiction" process [59, 60], and the predictions generated for time steps from $t-W+1$ to $t$ are "postdictions" of the past, rather than predictions in a literal sense.

\section{Parameter settings for the experiments}

The number of input, mean, and variance neurons was $N_{\mathrm{I}}=N_{\mathrm{M}}=N_{\mathrm{V}}=10$, respectively, corresponding to the dimensions of the robot's sensory states, and the number of PB neurons was $N_{\mathrm{P}}=2$. The number of context neurons was $N_{\mathrm{C}}=50$ and their time constant was $\tau_{i}=4$. For learning, the weights of the synaptic connections $w_{i j}\left(j \in I_{\mathrm{I}}, I_{\mathrm{C}}\right)$ were initialized with random values that follow uniform distributions on the intervals $\left[-\frac{1}{N_{\mathrm{I}}}, \frac{1}{N_{\mathrm{I}}}\right]\left(j \in I_{\mathrm{I}}\right)$, and $\left[-\frac{1}{N_{\mathrm{C}}}, \frac{1}{N_{\mathrm{C}}}\right]\left(j \in I_{\mathrm{C}}\right)$. Biases $b_{i}\left(I \in I_{\mathrm{C}}, I_{\mathrm{M}}, I_{\mathrm{V}}\right)$ were initialized with random values following a uniform distribution on the interval $[-1,1]$, and the internal states of PB neurons were initialized to 0 . These parameters were updated 100, 000 times during learning. In the adaptation phase, the internal states of the PB neurons were updated 10 times at each time step for a time window of length $W=10$.

\section{Evaluation of robot behavior}

To evaluate whether the robot's behavior in the adaptation phase was appropriate, time-series of sensory states generated in the experiment were compared with the target training data. A simple way to compare two time-series is to calculate the distances between values at corresponding time steps within a certain time window. However, this 
method is not necessarily appropriate for comparing the general characteristics of time-series because a phase shift will increase the distance between the series, even when the series is appropriate. To address this, we characterized the generated sequences as probability distributions of sensory states by compressing the time-series data in the time axis direction and using Kullback-Leibler (KL) divergence for comparison. In the evaluation, the probability distribution of each sensory state was found, with the one-dimensional space $[-1,1]$ divided into $N_{\text {bin }}=50$ subspaces. Using the acquired probability distributions, we calculated the KL divergence between the distribution of a certain sensory state in the experiment and the distribution of the corresponding sensory state in the target data for each sensory state. Then, the sum of the KL divergences over all sensory states was calculated and taken as a similarity score. The robot's action was judged as successful if the similarity score for joint angle was less than a threshold $\xi$, set here as 0.4 times the minimum similarity score between each pair of training data.

$$
S S(p \| q)=\sum_{k \in I_{\mathrm{M}}} D_{\mathrm{KL}}\left(p_{y_{k}} \| q_{y_{k}}\right)
$$

$$
S S(p \| q)<\xi=0.4 \times \min _{q_{i} \in U_{L}, q_{j} \in U_{R} \vee q_{i} \in U_{R}, q_{j} \in U_{L}} S S\left(q_{i} \| q_{j}\right)
$$

Here, $S S(p \| q)$ is the similarity score between the probability distribution of sensory states in experiment $p$ and the probability distribution in $\operatorname{target}$ data $q$; and $U_{L}$ and $U_{R}$ are sets of the probability distributions of sensory states in training data for left and right ball-pass behavior, respectively. The above method was used to evaluate the robot's performance both before environmental change (time steps 0-100) and after environmental change (time steps 200-300).

For the robot's behavior after environmental change (time steps 200-300), we also evaluated whether the networks could generate appropriate behavior without a change in PB activity in order to evaluate the influence of PB activity (higher-level prediction) on the generated behaviors. Specifically, the networks were required to generate temporal sequences by the "closed-loop" method using PB activity at time step 100 (the last time step before environmental change), where the initial two sensory inputs were given from the target training data. The idea is that if the neural network can successfully generate an appropriate visuomotor sequence using PB activity at time step 100, then the influence of this activity on behavior switching can be considered very weak. The performance was evaluated by comparing the time-series generated by the networks with the target training data by the above method. Here, the similarity score calculated for the robot's behavior after environmental change is denoted by $S S_{a c t i o n}$, and the similarity score calculated for time-series generated by the closed-loop method is denoted by $S S_{\text {reactivity }}$. Based on the two criterion, the robot's behaviors observed after environmental change were classified into one of the following four types: "proactive switching" (if $S S_{\text {action }}<\xi$ and $S S_{\text {reactivity }} \geqq \xi$ ), "reactive switching" (if $S S_{\text {action }}<\xi$ and $S S_{\text {reactivity }}<\xi$ ), "perseverative error" (if $S S_{\text {action }} \geqq \xi$ and $S S_{\text {reactivity }} \geqq \xi$ ), and "non-perseverative error" (if $S S_{\text {action }} \geqq \xi$ and $S S_{\text {reactivity }}<\xi$ ). Here, proactive switching indicates successful behavior as a result of updating higher-level activity (prediction) while reactive switching means an overtly 
successful behavior with a weak influence of higher-level activity. Perseverative error means a failure due to a deficit in switching higher-level activity while non-perseverative error means a failure due to an irrelevant change in higher-level activity.

\section{Simulation of functional disconnection}

Functional disconnection through the learning process in the hierarchical network was simulated by adding slight random noise to synaptic weights every time the weights were updated in the learning phase as follows:

$$
w_{i j}^{d i s}=w_{i j}+U\left(k\left|w_{i j}\right|\right) .
$$

Here, $i \in I_{\mathrm{C}}$ and $j \in I_{\mathrm{P}}$ for long-range disconnection, and $i \in I_{\mathrm{C}}$ and $j \in I_{\mathrm{C}}$ for local disconnection. $U\left(k\left|w_{i j}\right|\right)$ is noise following a uniform distribution on the interval $\left[-k\left|w_{i j}\right|, k\left|w_{i j}\right|\right] . k$ is a parameter determining the severity of functional disconnection: $k_{\text {long }}(\in\{0,0.2,0.4\})$ for long-range disconnection and $k_{\text {local }}(\in\{0,0.015,0.03\})$ for local disconnection. $k_{l o n g}=0$ and $k_{l o c a l}=0$ are the normal condition without disconnection. We obtained results from 10 trained networks with different initial synaptic weights for each setting of $k$. Random noise was added as one of the simplest implementations of reduced synaptic efficacy (functional disconnection).

\section{Statistical analysis}

In all statistical analyses, we used one-way ANOVA followed by the non-paired Shaffer test. All statistical tests were two-tailed, and the significance level was set at $p<0.05$. No statistical methods were used for determining the sample size in advance. Data analysis was conducted using R software (version 3.3.2).

\section{Data and code availability}

All data supporting the results are available within the manuscript and supplementary information files. Computer codes used in the learning and robot experiments are available online (https://ogata-lab.jp/projects/cognitive-robotics-group.html).

\section{Acknowledgments}

This work was supported by a MEXT Grant-in-Aid for Scientific Research on Innovative Areas, "Constructive Developmental Science" (No. JP24119003), a JSPS Grant-in-Aid for JSPS Research Fellow (No. JP19J20281), JSPS KAKENHI grants (Nos. JP17K12754, JP18KT0021, JP19K20364, JP19H04998, JP20H00001, and JP20H00625), JST CREST grants (Nos. JPMJCR16E2 and JPMJCR15E3), and the Program for Leading Graduate Schools, "Graduate Program for Embodiment Informatics" of the Ministry of Education, Culture, Sports, Science and Technology, Japan. 
466

467

468

469

470

\section{Author contributions}

H.I. conceived the study, performed the experiment, and analyzed the data. H.I., S.M., Y.Y., and T.O. designed the experiment and analysis, and wrote the paper.

\section{Competing interests}

The authors declare no competing interests. 
a

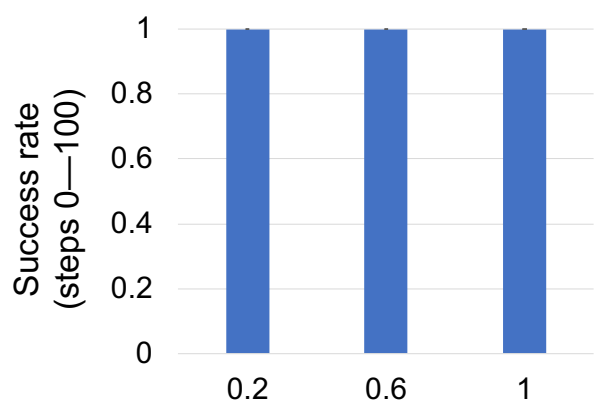

Ratio of synaptic connection

C

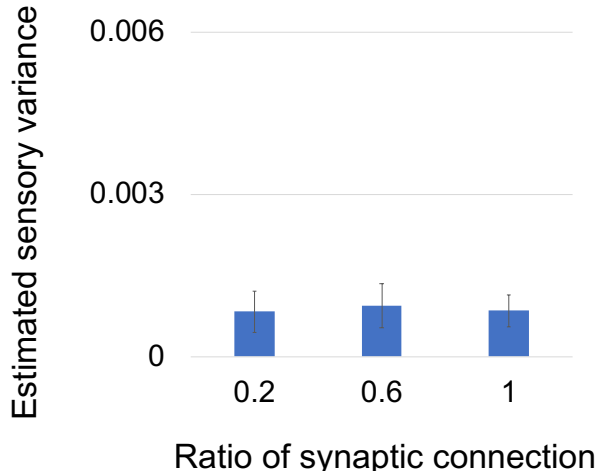

b

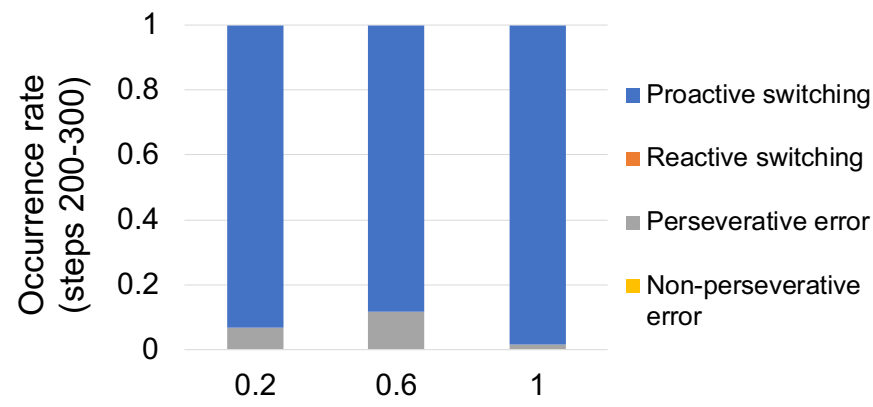

Ratio of synaptic connection

d

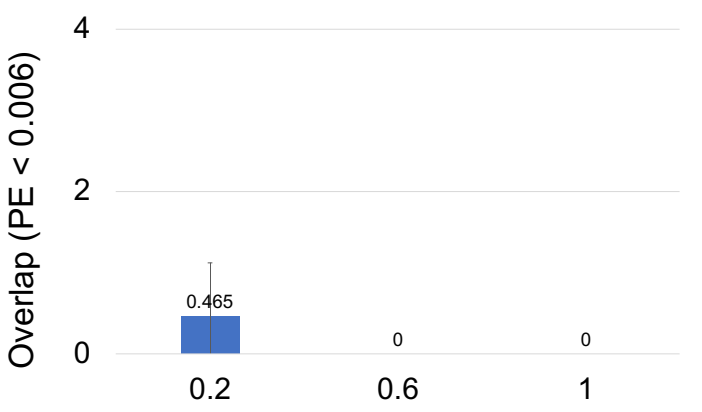

Ratio of synaptic connection

Supplementary Figure 1: Effects of anatomical reduction of long-range connections. (a) Success rates in the ball-pass interaction before environmental change (time steps 0-100) in the adaption phase. Under all conditions, the robot showed perfect performance. (b) Occurrence rates of each behavior type after environmental change (time steps 200-300) in the adaptation phase. The frequency of proactive switching was not significantly different among the three network conditions $(F(2,27)=1.40, p=0.26$, one-way ANOVA). (c) Estimated sensory variance during environmental change (time steps 100-200) in the adaptation phase. The values are averaged over dimensions of vision and time steps. The estimated sensory variance was not significantly different among the three network conditions $(F(2,27)=0.24, p=0.79$, one-way ANOVA). (d) Overlap of higher-level neural activities where calculated training errors for both left and right ball-pass behaviors were lower than 0.006. The overlap area was significantly different among the three network conditions $(F(2,27)=4.51, p=0.020$, one-way ANOVA). According to the post hoc Shaffer test, an $80 \%$ reduction in long-range connections led to a significant increase in overlap area compared with a $40 \%$ reduction $(p=0.015)$ and the normal condition (full connection) $(p=0.015)$. This suggests that excessive reduction in long-range connections could less the precision of higher-level prediction, although the alteration alone did not have a large impact on behavior-level performance. Reported values are the mean \pm SD for 10 trained networks 
a

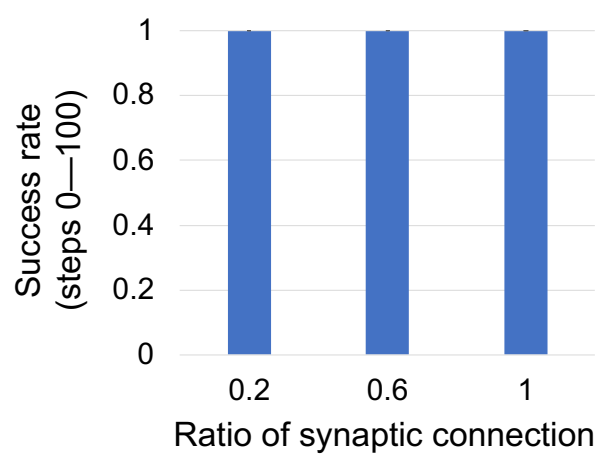

C

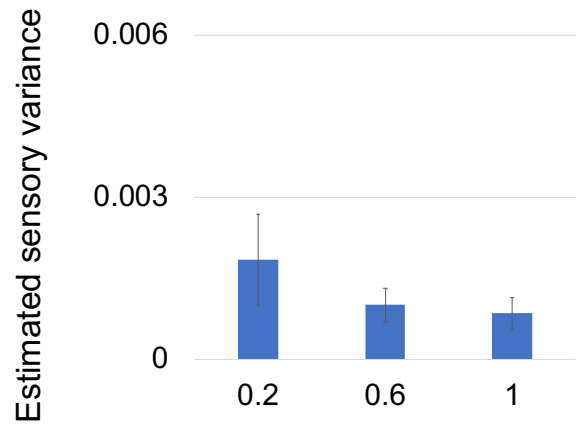

Ratio of synaptic connection b

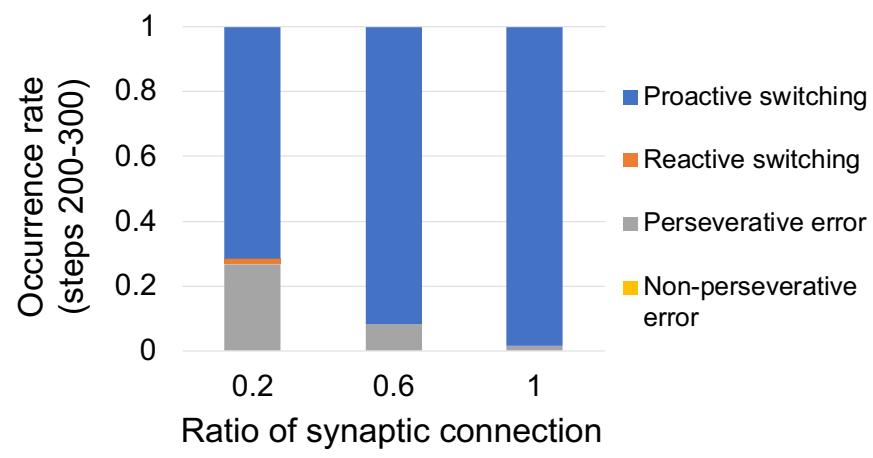

d

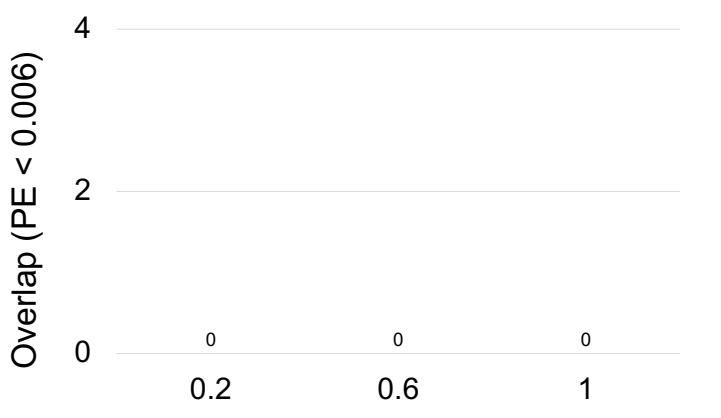

Ratio of synaptic connection

Supplementary Figure 2: Effects of simulating anatomical reduction of local connections. (a) Success rates in the ball-pass interaction before environmental change (time steps 0-100) in the adaption phase. Under all conditions, the robot showed perfect performance. (b) Occurrence rates of each behavior after environmental change (time steps 200-300) in the adaptation phase. The frequency of perseverative error was significantly different among the three network conditions $(F(2,27)=4.31, p=0.024$, oneway ANOVA). According to the post hoc Shaffer test, the frequency of perseverative error for an $80 \%$ reduction was significantly higher than that for a $40 \%$ reduction $(p=0.047)$ and the normal condition (full connection; $p=0.0086$ ). (c) Estimated sensory variance during environmental change (time steps 100-200) in the adaptation phase. Values are averaged over dimensions of vision and time steps. Estimated sensory variance was significantly different among the three network conditions $(F(2,27)=8.75$, $p=0.0012$, one-way ANOVA). According to the post hoc Shaffer test, an $80 \%$ reduction in local connections significantly increased the estimated sensory variance compared with a 40\% reduction $(p=0.0029)$ and the normal condition (full connection; $p<0.001$ ). These findings suggest that an excessive reduction in local connections could lead to sensory-ignoring behavior due to low sensory precision. (d) Overlap of higher-level neural activity where calculated training errors for both left and right ball-pass behaviors were lower than 0.006 . No overlap was observed under any conditions. Reported values are the mean \pm SD for 10 trained networks. 


\section{References}

[1] Feczko, E. et al. The heterogeneity problem: Approaches to identify psychiatric subtypes. Trends Cogn. Sci. 23, 584-601 (2019).

[2] Lichtenstein, P., Carlström, E., Råstam, M., Gillberg, C. \& Anckarsäter, H. The genetics of autism spectrum disorders and related neuropsychiatric disorders in childhood. Am. J. Psychiatry 167, 1357-1363 (2010).

[3] Cross-Disorder Group of the Psychiatric Genomics Consortium. Identification of risk loci with shared effects on five major psychiatric disorders: a genome-wide analysis. Lancet 381, 1371-1379 (2013).

[4] Craig, F. et al. Overlap between autism spectrum disorders and attention deficit hyperactivity disorder: Searching for distinctive/common clinical features. Autism Res. 8, 328-337 (2015).

[5] De Crescenzo, F. et al. Autistic symptoms in schizophrenia spectrum disorders: A systematic review and meta-analysis. Front. Psychiatry 10, 1-11 (2019).

[6] Cicchetti, D. \& Rogosch, F. A. Equifinality and multifinality in developmental psychopathology. Dev. Psychopathol. 8, 597-600 (1996).

[7] Huys, Q. J. M., Maia, T. V. \& Frank, M. J. Computational psychiatry as a bridge from neuroscience to clinical applications. Nat. Neurosci. 19, 404-413 (2016).

[8] American Psychiatric Association. Diagnostic and Statistical Manual of Mental Disorders (American Psychiatric Association, Washington, DC, 2013).

[9] Levit-Binnun, N., Davidovitch, M. \& Golland, Y. Sensory and motor secondary symptoms as indicators of brain vulnerability. J. Neurodev. Disord. 5, 26 (2013).

[10] Marco, E. J., Hinkley, L. B. N., Hill, S. S. \& Nagarajan, S. S. Sensory processing in autism: a review of neurophysiologic findings. Pediatr. Res. 69, 48R-54R (2011).

[11] Zwaigenbaum, L. et al. Behavioral manifestations of autism in the first year of life. Int. J. Dev. Neurosci. 23, $143-152$ (2005).

[12] Gliga, T., Jones, E. J. H., Bedford, R., Charman, T. \& Johnson, M. H. From early markers to neuro-developmental mechanisms of autism. Dev. Rev. 34, 189-207 (2014).

[13] Ben-Sasson, A., Soto, T. W., Heberle, A. E., Carter, A. S. \& Briggs-Gowan, M. J. Early and concurrent features of ADHD and sensory over-responsivity symptom clusters. J. Atten. Disord. 21, 835-845 (2017).

[14] Wolff, J. J. et al. A longitudinal study of parent-reported sensory responsiveness in toddlers at-risk for autism. J. Child Psychol. Psychiatry 60, 314-324 (2019). 
[15] Gonzalez-Gadea, M. L. et al. Predictive coding in autism spectrum disorder and attention deficit hyperactivity disorder. J. Neurophysiol. 114, 2625-2636 (2015).

[16] Haker, H., Schneebeli, M. \& Stephan, K. E. Can Bayesian theories of autism spectrum disorder help improve clinical practice? Front. Psychiatry 7, 107 (2016).

[17] Clark, A. Surfing Uncertainty: Prediction, Action, and the Embodied Mind (Oxford University Press, New York, 2016).

[18] Keller, G. B. \& Mrsic-Flogel, T. D. Predictive processing: A canonical cortical computation. Neuron 100, 424-435 (2018).

[19] Pellicano, E. \& Burr, D. When the world becomes 'too real': a Bayesian explanation of autistic perception. Trends Cogn. Sci. 16, 504-510 (2012).

[20] Lawson, R. P., Rees, G. \& Friston, K. J. An aberrant precision account of autism. Front. Hum. Neurosci. 8, 302 (2014).

[21] Van de Cruys, S. et al. Precise minds in uncertain worlds: predictive coding in autism. Psychol. Rev. 121, 649-675 (2014).

[22] Palmer, C. J., Lawson, R. P. \& Hohwy, J. Bayesian approaches to autism: Towards volatility, action, and behavior. Psychol. Bull. 143, 521-542 (2017).

[23] Just, M. A., Cherkassky, V. L., Keller, T. A. \& Minshew, N. J. Cortical activation and synchronization during sentence comprehension in high-functioning autism: evidence of underconnectivity. Brain 127, 1811-1821 (2004).

[24] Kana, R. K., Keller, T. A., Cherkassky, V. L., Minshew, N. J. \& Just, M. A. Atypical frontal-posterior synchronization of Theory of Mind regions in autism during mental state attribution. Soc. Neurosci. 4, 135-152 (2009).

[25] Mazaheri, A. et al. Functional disconnection of frontal cortex and visual cortex in attention-deficit/hyperactivity disorder. Biol. Psychiatry 67, 617-623 (2010).

[26] Just, M. A., Keller, T. A., Malave, V. L., Kana, R. K. \& Varma, S. Autism as a neural systems disorder: A theory of frontal-posterior underconnectivity. Neurosci. Biobehav. Rev. 36, 1292-1313 (2012).

[27] Goto, Y., Yang, C. R. \& Otani, S. Functional and dysfunctional synaptic plasticity in prefrontal cortex: Roles in psychiatric disorders. Biol. Psychiatry 67, 199-207 (2010).

[28] Friston, K., Brown, H. R., Siemerkus, J. \& Stephan, K. E. The dysconnection hypothesis (2016). Schizophr. Res. 176, 83-94 (2016). 
[29] Geschwind, D. H. \& Levitt, P. Autism spectrum disorders: developmental disconnection syndromes. Curr. Opin. Neurobiol. 17, 103-111 (2007).

[30] Dinstein, I. et al. Disrupted neural synchronization in toddlers with autism. Neuron 70, 1218-1225 (2011).

[31] Keehn, B., Wagner, J. B., Tager-Flusberg, H. \& Nelson, C. A. Functional connectivity in the first year of life in infants at-risk for autism: a preliminary near-infrared spectroscopy study. Front. Hum. Neurosci. 7, 444 (2013).

[32] Righi, G., Tierney, A. L., Tager-Flusberg, H. \& Nelson, C. A. Functional connectivity in the first year of life in infants at risk for autism spectrum disorder: an EEG study. PLoS ONE 9, e105176 (2014).

[33] Brown, H., Adams, R. A., Parees, I., Edwards, M. \& Friston, K. Active inference, sensory attenuation and illusions. Cogn. Process. 14, 411-427 (2013).

[34] Rosenberg, A., Patterson, J. S. \& Angelaki, D. E. A computational perspective on autism. Proc. Natl. Acad. Sci. U. S. A. 112, 9158-9165 (2015).

[35] Anticevic, A., Murray, J. D. \& Barch, D. M. Bridging levels of understanding in schizophrenia through computational modeling. Clin. Psychol. Sci. 3, 433-459 (2015).

[36] Yamashita, Y. \& Tani, J. Spontaneous prediction error generation in schizophrenia. PLoS ONE 7, e37843-e37843 (2012).

[37] Idei, H. et al. A neurorobotics simulation of autistic behavior induced by unusual sensory precision. Comput. Psychiatr. 2, 164-182 (2018).

[38] Lewis, M., Fineberg, N. \& Cañamero, L. A robot model of oc-spectrum disorders: design framework, implementation and first experiments. Comput. Psychiatr. 3, 40-75 (2019).

[39] Murata, S., Namikawa, J., Arie, H., Sugano, S. \& Tani, J. Learning to reproduce fluctuating time series by inferring their time-dependent stochastic properties: Application in robot learning via tutoring. IEEE Trans. Autono. Mental Develop. 5, 298-310 (2013).

[40] Tani, J., Ito, M. \& Sugita, Y. Self-organization of distributedly represented multiple behavior schemata in a mirror system: reviews of robot experiments using RNNPB. Neural Netw. 17, 1273-1289 (2004).

[41] Shaffer, J. P. Modified sequentially rejective multiple test procedures. J. Am. Stat. Assoc. 81, 826-831 (1986).

[42] Penzes, P., Cahill, M. E., Jones, K. A., VanLeeuwen, J.-E. \& Woolfrey, K. M. Dendritic spine pathology in neuropsychiatric disorders. Nat. Neurosci. 14, 285 (2011).

[43] Frith, C. Is autism a disconnection disorder? Lancet Neurol. 3, 577 (2004).

[44] Lainhart, J. E. et al. Macrocephaly in children and adults With autism. J. Am. Acad. Child Adolesc. Psychiatry 36, 282-290 (1997). 
[45] Tang, G. et al. Loss of mTOR-dependent macroautophagy causes autistic-like synaptic pruning deficits. Neuron 83, 1131-1143 (2014).

[46] Thomas, M. S. C., Davis, R., Karmiloff-Smith, A., Knowland, V. C. P. \& Charman, T. The over-pruning hypothesis of autism. Dev. Sci. 19, 284-305 (2016).

[47] Zwaigenbaum, L. et al. Early head growth in infants at risk of autism: A baby siblings research consortium study. J. Am. Acad. Child Adolesc. Psychiatry 53, 1053-1062 (2014).

[48] Corlett, P. R. et al. Hallucinations and strong priors. Trends Cogn. Sci. 23, 114-127 (2019).

[49] Sterzer, P. et al. The predictive coding account of psychosis. Biol. Psychiatry 84, 634-643 (2018).

[50] Ben-Sasson, A. et al. Sensory clusters of toddlers with autism spectrum disorders: differences in affective symptoms. J. Child Psychol. Psychiatry 49, 817-825 (2008).

[51] Ben-Sasson, A. et al. A meta-analysis of sensory modulation symptoms in individuals with autism spectrum disorders. J. Autism Dev. Disord. 39, 1-11 (2009).

[52] Baranek, G. T. et al. Hyporesponsiveness to social and nonsocial sensory stimuli in children with autism, children with developmental delays, and typically developing children. Dev. Psychopathol. 25, 307-320 (2013).

[53] Khan, S. et al. Local and long-range functional connectivity is reduced in concert in autism spectrum disorders. Proc. Natl. Acad. Sci. U. S. A. 110, 3107-3112 (2013).

[54] Dajani, D. R. \& Uddin, L. Q. Local brain connectivity across development in autism spectrum disorder: A cross-sectional investigation. Autism Res. 9, 43-54 (2016).

[55] Friston, K. The free-energy principle : a unified brain theory? Nat. Rev. Neurosci. 11, 127-138 (2010).

[56] Fetz, E. E. \& Shupe, L. E. Recurrent network: neurophysiological modeling (MIT Press, Cambridge, MA, 2002).

[57] Yamashita, Y. \& Tani, J. Emergence of functional hierarchy in a multiple timescale neural network model: A humanoid robot experiment. PLoS Comput. Biol. 4, e1000220 (2008).

[58] Rumelhart, D. E., Hinton, G. E. \& Williams, R. J. Learning representations by back-propagating errors. Nature 323, 533-536 (1986).

[59] Eagleman, D. M. \& Sejnowski, T. J. Motion integration and postdiction in visual awareness. Science 287, 2036-2038 (2000).

[60] Shimojo, S. Postdiction: its implications on visual awareness, hindsight, and sense of agency. Front. Psychol. 5, 196 (2014). 